\title{
Biologia reprodutiva de Pachyurus bonariensis (Perciformes, Sciaenidae) na fase pré-represamento do arroio Taquarembó, Sul do Brasil
}

\author{
Giovana I. Lagemann ${ }^{1} \&$ Clarice B. Fialho ${ }^{2}$
}

1. Instituto Federal de Educação, Ciência e Tecnologia Sul-rio-grandense, Av. das Indústrias, 1865 - Bairro Universitário, 95800-000, Venâncio Aires, RS, Brasil. (giovanail@ibest.com.br) 2. Universidade Federal do Rio Grande do Sul, Departamento de Zoologia, Laboratório de Ictiologia, Av. Bento Gonçalves, 9500, bloco IV, prédio 43435, 91501-970 Porto Alegre, RS, Brasil. (cbfialho@via-rs.net)

\begin{abstract}
Reproductive biology of Pachyurus bonariensis (Perciformes, Sciaenidae) before the daming of the Taquarembó stream in southern Brazil. This work describes aspects of the reproductive biology of Pachyurus bonariensis (Steindachner, 1879) through the analysis of 278 specimens collected from July 2006 to July 2007. The estimated reproductive period lasted from October 2006 to February 2007 and showed a positive correlation with photoperiod and water conductivity. The oocyte development was synchronous in more than two groups, characterizing the species as multiple spawner. Mean absolute fecundity was 39,313 oocytes and relative fecundity was 0.43 oocysts per milligram of total weight. The length of the first specimen mature was set at $112.6 \mathrm{~mm}$ for females and males $110.7 \mathrm{~mm}$ and sex ratio of the population as a whole did not differ significantly from $1: 1$.
\end{abstract}

KEYWORDS. Croaker, oocytes development, fecundity.

RESUMO. Este trabalho descreve aspectos da biologia reprodutiva de Pachyurus bonariensis (Steindachner, 1879) através da análise de 278 exemplares coletados de julho de 2006 a julho de 2007. O período reprodutivo estimado estendeu-se de outubro de 2006 a fevereiro de 2007 e mostrou uma correlação positiva com o fotoperíodo e a condutividade da água. O desenvolvimento oocitário foi sincrônico em mais de dois grupos, caracterizando uma desova do tipo parcelada. A fecundidade média absoluta foi de 39.313 oócitos e a fecundidade relativa foi de 0,43 oócitos por miligrama de peso total. O comprimento do primeiro indivíduo maduro foi estabelecido em 112,6 mm para as fêmeas e em 110,7 mm para os machos e a proporção sexual da população como um todo não diferiu significativamente de 1:1.

PALAVRAS-CHAVE. Corvina, desenvolvimento oocitário, fecundidade.

Pachyurus bonariensis (Steindachner, 1879) é uma espécie de peixe da família Sciaenidae conhecida popularmente como corvina-de-rio, curvina ou maria-luiza. A distribuição original da espécie inclui os rios do sistema Paraná-Paraguai-Uruguai, à jusante do reservatório de Itaipu, no Brasil, Paraguai, Uruguai e Argentina (CASATTI, 2001). Desde 2001 a espécie passou a ser registrada também no sistema da laguna dos Patos, sendo considerada alóctone (não nativa) nesse sistema (Dufech \& FialHo, 2007). Estudos mais recentes demonstram que $P$. bonariensis se encontra amplamente distribuída e adaptada à bacia da laguna dos Patos (Dufech \& Fialho, 2007; SAccol-Pereira \& Fialho, 2010); também está muito bem distribuída e adaptada ao sistema da laguna dos Patos, inclusive passando a fazer parte do estoque de importância comercial na lagoa do Casamento, que pertencente a esse sistema (Milani \& Fontoura, 2007). Durante o estudo da comunidade de peixes do arroio Taquarembó, tributário do rio Santa Maria, sistema do rio Uruguai, no Sul do Brasil, entre 2006 e 2007, P. bonariensis foi considerada uma das espécies mais abundantes (C. E. Machado, dados inéditos). O arroio Taquarembó é um afluente da margem esquerda do rio Santa Maria, bacia do rio Uruguai, na fronteira sudoeste do estado do Rio Grande do Sul. A construção da Barragem do Arroio Taquarembó, foi projetada com o objetivo de aumentar, principalmente, a oferta de água para consumo humano. Conforme Cecilıo et al. (1997) a construção de represamentos interceptando cursos de água naturais tem aumentado de forma significativa nos últimos anos, com a ictiofauna desses sistemas sofrendo, nesse processo, profundas alterações. Segundo Agostinho et al. (1992), esses empreendimentos produzem, tanto à montante como à jusante, consequências inevitáveis como alterações na composição específica e na estrutura das comunidades de peixes nativos.

O conhecimento sobre a ictiofauna pré e pósbarramentos é muito importante, pois permite compreender melhor a dinâmica das populações e suas possíveis alterações biológicas e ecológicas ao longo da transição ambiente lótico - lêntico. Para P. bonariensis, poucos são os trabalhos com esse enfoque (FLORES \& HiRT, 2002; PACHeCo et al., 2008). O objetivo desse estudo foi descrever os aspectos da biologia reprodutiva de P. bonariensis antes da construção do barramento no arroio Taquarembó, bacia do rio Santa Maria, sistema do rio Uruguai, no sul do Brasil e, com isso, contribuir com dados básicos para eventuais ações de manejo e conservação dessa espécie.

\section{MATERIAL E MÉTODOS}

Os espécimes foram coletados mensalmente no arroio Taquarembó (3048’19’'S 54³5’01’'W), bacia hidrográfica do rio Santa Maria, município de Dom Pedrito, sul do Brasil, no período de julho de 2006 a julho de 2007, com exceção dos meses de agosto de 2006 e junho de 2007, onde as condições metereológicas não permitiram 
a realização de coletas. Foram utilizadas redes de espera com malhas 1,5; 2,5; 3,5 e 5,0 cm entre nós adjacentes, com $10 \mathrm{~m}$ de comprimento por $1,5 \mathrm{~m}$ de altura. Os peixes foram submetidos a eutanásia com eogenol (óleo de cravo) e, posteriormente, fixados, em campo, em solução de formalina $10 \%$, sendo posteriormente transferidos para etanol $70^{\circ} \mathrm{GL}$. Dados abióticos como temperatura da água, oxigênio dissolvido e condutividade foram registrados no momento da coleta. O fotoperíodo foi obtido pelo programa Skymap e os dados de pluviosidade obtidos junto ao $8^{\circ}$ Distrito de Meteorologia de Porto Alegre, RS. De todos os exemplares coletados, foram tomados os dados biométricos tais como comprimento padrão (Lp), em mm, e peso total $(\mathrm{Wt})$, peso das gônadas $(\mathrm{Wg})$ e do estômago (We), em gramas. Com os dados obtidos foram calculados o índice gonadossomático (IGS=(Wg/Wt).100) (VAzZoler, 1996) e o índice de repleção (IR=(We/Wt).100) (SANTOS,1978). As gônadas foram classificadas macroscopicamente quanto ao grau de maturação conforme VAzzoLER (1996). O período reprodutivo foi estabelecido através da análise da variação mensal dos valores médios dos índices gonadossomáticos (IGS) e pela distribuição das frequências relativas dos estádios de maturação gonadal. Para verificar possíveis correlações entre o Índice Gonadossomático (IGS) e o Índice de Repleção (IR) foi utilizado o teste estatístico não paramétrico de Spearman (ZAR, 1999) e para avaliar se havia influência dos fatores abióticos (temperatura da água, condutividade, fotoperíodo e pluviosidade) na variação dos valores médios de IGS, foi utilizado o teste de regressão múltipla (ZAR, 1999).

Foram selecionados 11 ovários no estádio maduro, com os maiores valores de IGS, para cálculo da fecundidade média absoluta, empregando-se o método gravimétrico sugerido por VAzzoLER (1996). A fecundidade relativa foi estimada conforme ADEBISI (1987), calculando-se o número de oócitos por miligrama de peso da fêmea. Para analisar o tipo de desenvolvimento oocitário, foram selecionadas 404 gônadas, representando os estádios em maturação (15), maduro (11), semiesgotado (9) e esgotado (9) e, de cada uma, retirou-se uma subamostragem de 150 oócitos com o auxílio de uma pipeta. Em estereomicroscópio com ocular milimetrada, o diâmetro máximo desses oócitos foi medido, obtendo-se com isso a frequência de oócitos por classes de diâmetro segundo VAzzoLER (1996). O tamanho da primeira maturação foi estimado utilizando-se o método proposto por SATo \& Godinho (1988), que considera o tamanho de primeira maturação como o menor tamanho onde ocorrem indivíduos em estádio avançado de maturação gonadal. A existência ou não de diferenças significativas nas proporções de machos e fêmeas entre os meses e por classes de comprimento foi verificada aplicando-se o teste Qui-quadrado $\left(\chi^{2}\right)(\alpha=0,05)\left(Z_{A R}, 1999\right)$. Exemplares testemunhos foram catalogados na Coleção de Peixes do Departamento de Zoologia, Universidade Federal do Rio Grande do Sul (UFRGS 10306).

\section{RESULTADOS}

Foram analisados 126 machos e 152 fêmeas totalizando 278 espécimes, com comprimento-padrão entre $73,68 \mathrm{~mm}$ a $198,58 \mathrm{~mm}$ e $59,91 \mathrm{~mm}$ a $203,63 \mathrm{~mm}$, respectivamente.

A variação mensal dos valores médios do IGS indicou que o período reprodutivo para machos (Fig. 1) e fêmeas (Fig. 2) teve início em outubro de 2006 e estendeu-se até janeiro de 2007, compreendendo os meses correspondentes à primavera e ao verão no hemisfério sul.

Através de análises macroscópicas das gônadas foram definidos os seguintes estádios de maturação gonadal: imaturo, em maturação, maduro, semiesgotado e esgotado, sendo que, para os machos, não foi possível observar este último estádio. Na maioria dos estádios de maturação os ovários apresentavam-se túrgidos e compactos, independente do tamanho, exceto no estádio esgotado.

Machos em maturação ocorreram a partir do mês de abril e fêmeas, a partir do mês de maio. Machos maduros foram frequentes de setembro a janeiro, enquanto que as fêmeas somente de novembro a janeiro, corroborando os dados de IGS. Fêmeas semiesgotadas ocorreram nos meses de dezembro e janeiro e, para ambos os sexos, o estádio esgotado foi frequente a partir de fevereiro. $\mathrm{O}$ período de recrutamento de machos imaturos ocorreu de abril a novembro e de fêmeas imaturas ocorreu de março a outubro (Figs 3, 4).

O período reprodutivo também pode ser corroborado pela distribuição das médias de IGS de fêmeas por estádios de maturação (Fig. 5). Essa análise mostrou que o IGS das fêmeas em maturação elevou-se na primavera, com pico ocorrendo em outubro de 2006. De novembro de 2006 a janeiro de 2007 os maiores valores de IGS foram de fêmeas maduras, mas é possível verificar que, de dezembro de 2006 a janeiro de 2007 ocorreram paralelamente picos de IGS

Tab. I. Relação entre as médias mensais do índice gonadossomático (IGS) de machos e fêmeas de Pachyurus bonariensis (Steindachner, 1879) e os fatores abióticos (condutividade, oxigênio dissolvido e temperatura da água, fotoperíodo e pluviosidade) no arroio Taquarembó, bacia hidrográfica do rio Santa Maria, sul do Brasil [F, resultado do teste de Fisher; $(*)$ indica correlação significativa $(\mathrm{p}<0,05]$.

\begin{tabular}{lccc}
\hline $\begin{array}{l}\text { Índice gonadossomático } \\
\text { e dados abióticos }\end{array}$ & & $\mathrm{F}$ & $\mathrm{p}$ \\
\hline \multirow{2}{*}{ Condutividade } & machos & 11,581 & $0,008^{*}$ \\
& fêmeas & 7,252 & $0,025^{*}$ \\
Oxigênio dissolvido & machos & 0,003 & 0,961 \\
& fêmeas & 0,325 & 0,582 \\
Temperatura da água & machos & 1,493 & 0,253 \\
& fêmeas & 2,414 & 0,155 \\
Fotoperíodo & machos & 7,376 & $0,024^{*}$ \\
& fêmeas & 5,648 & $0,041^{*}$ \\
Pluviosidade & machos & 0,320 & 0,586 \\
& fêmeas & 0,608 & 0,455 \\
\hline
\end{tabular}




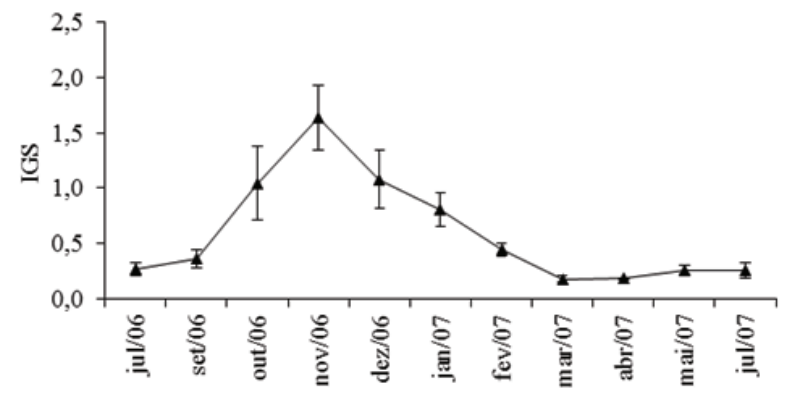

Fig. 1. Variação mensal do índice gonadossomático (IGS) médio ( \pm desvio padrão) para machos de Pachyurus bonariensis (Steindachner, 1879) do arroio Taquarembó, bacia hidrográfica do rio Santa Maria, sul do Brasil, no período de julho de 2006 a julho de 2007. Barras verticais representam o desvio padrão das médias.

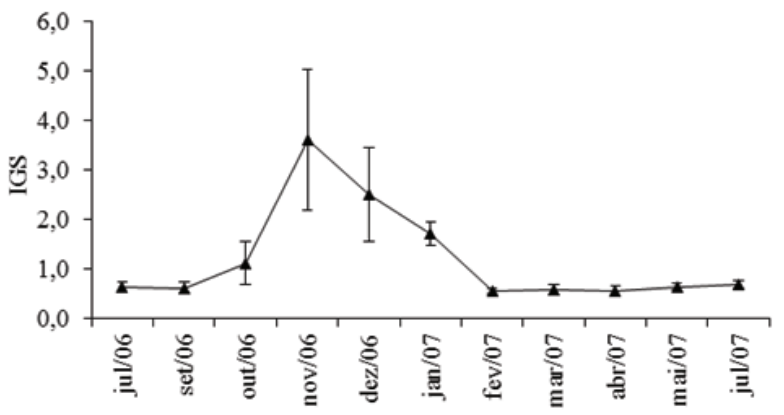

Fig. 2. Variaçäo mensai đo indiice gonađiossomático (İGS) médio ( \pm desvio padrão) para fêmeas de Pachyurus bonariensis (Steindachner, 1879) do arroio Taquarembó, bacia hidrográfica do rio Santa Maria, sul do Brasil, no período de julho de 2006 a julho de 2007. Barras verticais representam o desvio padrão das médias apresentadas.

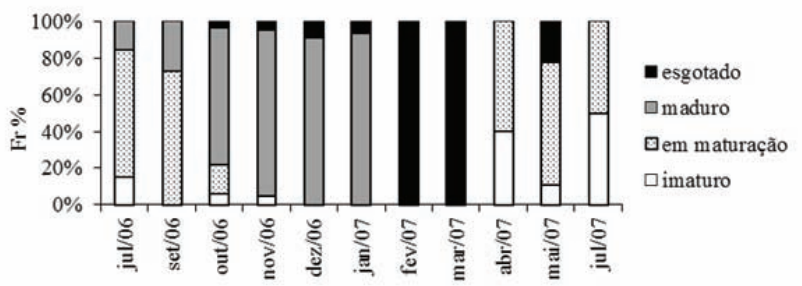

Fig. 3. Variação mensal das frequências relativas (percentual) dos estádios de maturação gonadal de machos de Pachyurus bonariensis (Steindachner, 1879) do arroio Taquarembó, bacia hidrográfica do rio Santa Maria, sul do Brasil, no período de julho de 2006 a julho de 2007.

de fêmeas semiesgotadas. A partir de fevereiro de 2007 o IGS registrado foi apenas de fêmeas no estádio esgotado permanecendo até julho de 2007. O reaparecimento de fêmeas em maturação ocorreu a partir de maio de 2007.

Não houve correlação significativa entre os valores de IGS e de IR ( $r=0,304$ e $\mathrm{p}=0,364$ para machos; $\mathrm{r}=$ 0,395 e $\mathrm{p}=0,220$ para as fêmeas), mas os maiores valores do índice de repleção foram atingidos em outubro, antes do pico reprodutivo (Fig. 6). Entre os fatores abióticos testados, apenas a condutividade e o fotoperíodo apresentaram relação significativa com o IGS $(\mathrm{p}<0,05)$ (Tab. I).

A distribuição da frequência absoluta dos diâmetros

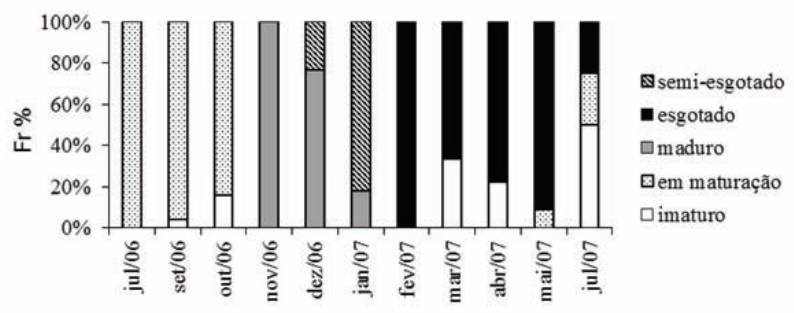

Fig. 4. Variação mensal das frequências relativas (percentual) dos estádios de maturação gonadal de fêmeas de Pachyurus bonariensis (Steindachner, 1879) do arroio Taquarembó, bacia hidrográfica do rio Santa Maria, região sul do Brasil no período de julho de 2006 a julho de 2007.

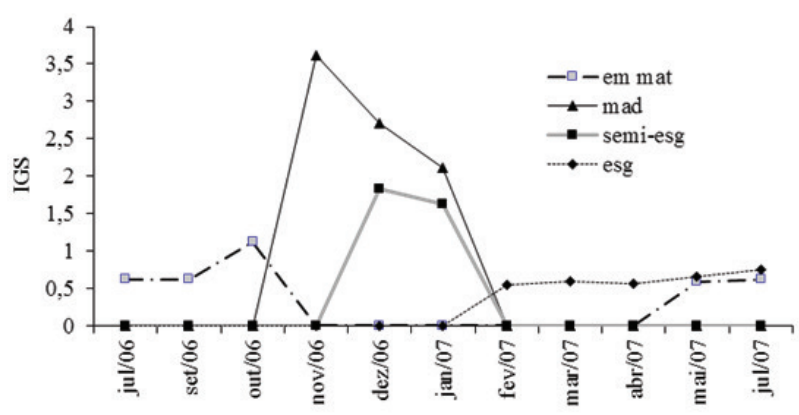

Fig. 5. Variação do índice gonadossomático médio (IGS) por estádios de maturação das fêmeas de Pachyurus bonariensis (Steindachner, 1879) do arroio Taquarembó, bacia hidrográfica do rio Santa Maria, sul do Brasil, no período de julho de 2006 a julho de 2007.

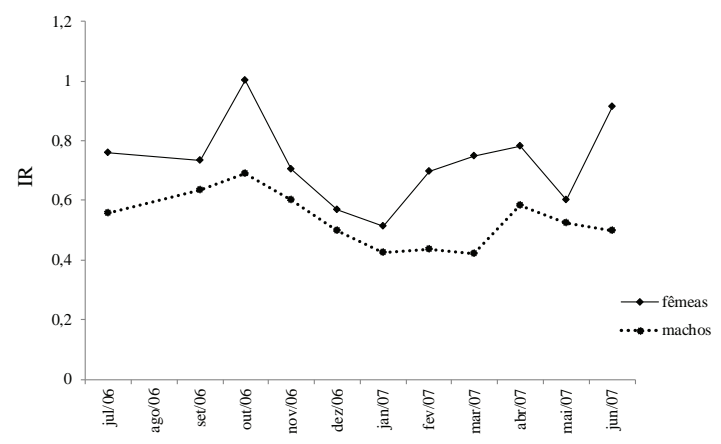

Fig. 6. Distribuição mensal dos valores médios do índice de repleção (IR) de machos e fêmeas de Pachyurus bonariensis (Steindachner, 1879) coletados no arroio Taquarembó, bacia hidrográfica do rio Santa Maria, sul do Brasil, entre julho de 2006 e julho de 2007.

dos oócitos vitelinados (Fig. 7) mostrou que a espécie possui desenvolvimento oocitário do tipo sincrônico em mais de dois grupos, sugerindo uma desova do tipo parcelada. Nessa distribuição, torna-se evidente a presença de um lote de oócitos de reserva em todos os estádios de maturação, bem como a existência de mais duas modas de oócitos nas gônadas maduras e semiesgotadas.

A fecundidade absoluta média foi de 39.313 oócitos ( $\pm 17.257 \mathrm{DP})$, com uma variação de 24.338 a 86.920 oócitos para fêmeas com comprimento-padrão de 147,28 a 192,31 $\mathrm{mm}$. A fecundidade relativa média variou de 0,34 a 0,60 oócitos/mg de peso total, com média de 0,43 oócitos/mg 

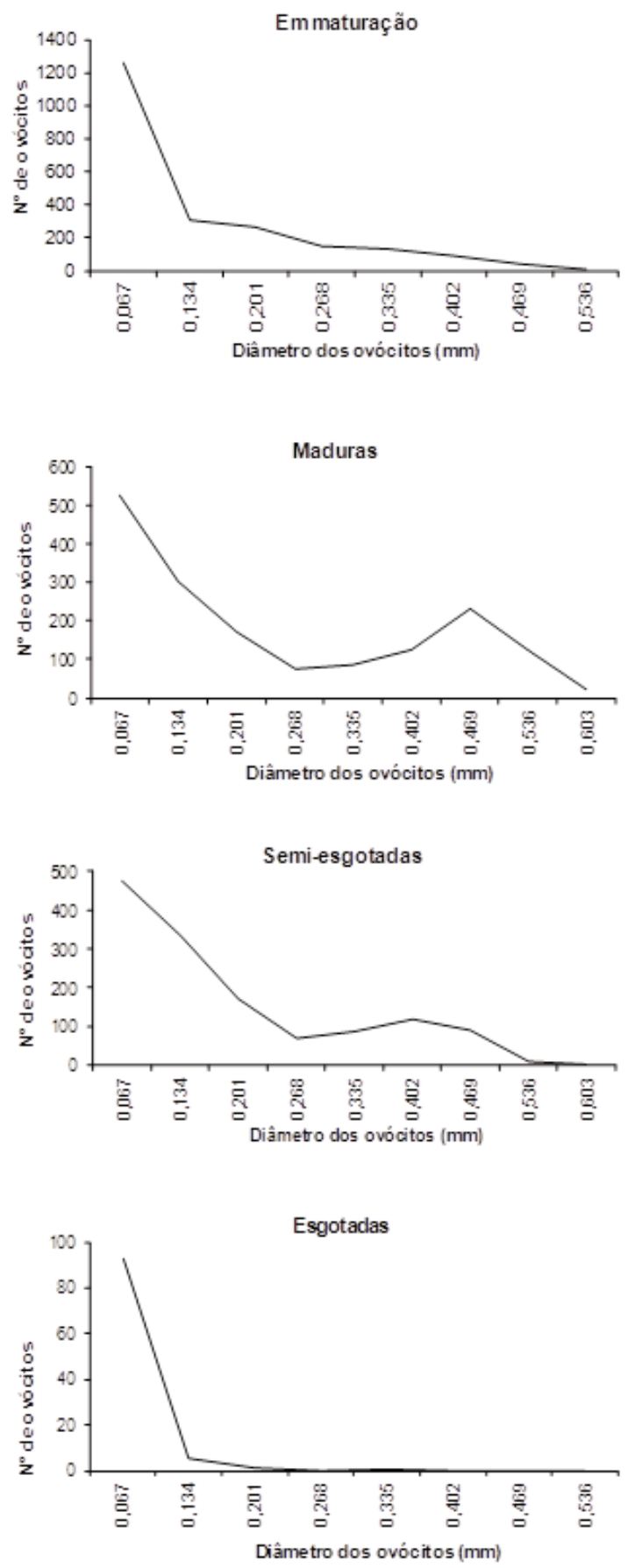

Fig. 7. Distribuição do número de oócitos por classes de diâmetro diâmetros nos diferentes estádios de maturação gonadal de fêmeas de Pachyurus bonariensis (Steindachner, 1879) do arroio Taquarembó na bacia hidrográfica do rio Santa Maria na região sul do Brasil no período de julho de 2006 a julho de 2007.

de peso total $( \pm 0,08 \mathrm{DP})$.

O tamanho da primeira maturação estimado para $P$. bonariensis no arroio Taquarembó, levando-se em consideração os menores indivíduos maduros, foi de 112,67 mm para fêmeas e de $110,71 \mathrm{~mm}$ para machos.

A proporção sexual total da população não diferiu significativamente de 1:1 $\left(\chi^{2}=2,43 ; p=0,1189\right)$. Diferenças

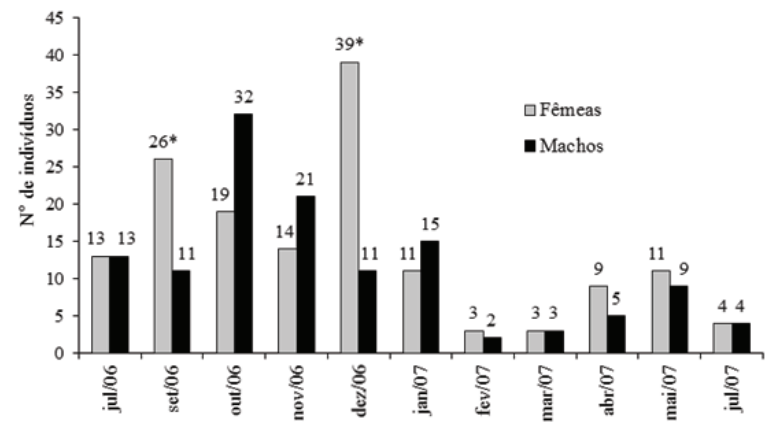

Fig. 8. Distribuição dos valores absolutos de machos e fêmeas de Pachyurus bonariensis (Steindachner, 1879) do arroio Taquarembó, bacia hidrográfica do rio Santa Maria, sul do Brasil, no período de julho de 2006 a julho de 2007. Asteriscos (*) indicam diferenças significativas na proporção sexual $\left(\chi^{2}>3,84\right)$.

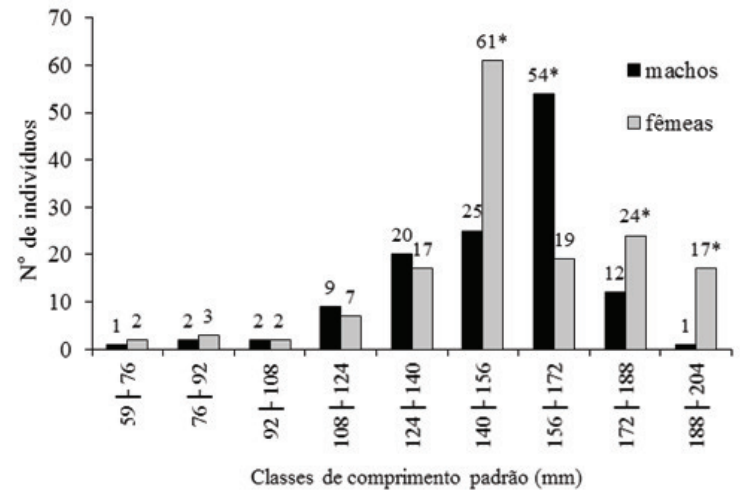

Fig. 9. Distribuição dos valores absolutos de machos e fêmeas de Pachyurus bonariensis (Steindachner, 1879) por classes de comprimento padrão no arroio Taquarembó, bacia hidrográfica do rio Santa Maria, sul do Brasil, no período de julho de 2006 a julho de 2007. Asteriscos (*) indicam diferenças significativas na proporção sexual $\left(\chi^{2}>3,84\right)$.

significativas na proporção sexual foram encontradas somente nos meses de setembro (26 fêmeas: 11 machos; $\chi^{2}=6,08 ; \mathrm{p}=0,0137$ ) e dezembro (39 fêmeas: 11 machos; $\chi^{2}=15,68 ; p=0,0001$ ) (Fig. 8).

Houve diferenças significativas na proporção sexual nas quatro maiores classes de comprimento. Houve predomínio de fêmeas nas classes $140-156 \mathrm{~mm}\left(\chi^{2}=15,07\right.$; $\mathrm{p}=0,0001), 172-188 \mathrm{~mm}\left(\chi^{2}=4,0 ; \mathrm{p}=0,0455\right)$ e $188-204$ $\mathrm{mm}\left(\chi^{2}=14,22 ; \mathrm{p}=0,0002\right)$. Apenas na classe $156-172 \mathrm{~mm}$ $\left(\chi^{2}=16,78 ; \mathrm{p}=0,0001\right)$ foram capturados mais machos do que fêmeas (Fig. 9).

\section{DISCUSSÃO}

No arroio Taquarembó, P. bonariensis se reproduziu durante a primavera e o verão e seu desenvolvimento reprodutivo coincidiu com o aumento do fotoperíodo. Esse é um dos fatores abióticos que modulam as táticas reprodutivas de muitas espécies (VAzzoler, 1996) e também relatado em outros estudos realizados nas regiões subtropical ou temperada no sul do Brasil (AzEvedo et al., 
2000; GonÇALVES et al., 2005; Muniz et al., 2008; Giora \& Fialho, 2009; AzEvedo, 2010). Estudos realizados com essa mesma espécie, como o de Flores \& HiRT (2002) na represa de Yacyretá na Argentina e o de MArques et al.(2007) no lago Guaíba e na laguna dos Patos, registraram que a atividade reprodutiva ocorreu nesse mesmo período do ano. Conforme SAntin (2009), na baía Sinhá Mariana, em Cuiabá, as maiores densidades de larvas dessa espécie ocorreram nos meses mais quentes do ano (primaveraverão) sugerindo que, na região central do Brasil, este também seja o principal período de reprodução de $P$. bonariensis.

Os machos iniciaram o processo de maturação antes das fêmeas e permaneceram em atividade reprodutiva por um período maior. Esse fenômeno, segundo NeUberger et al. (2007) e SANTOS et al. (2010) é comum a muitas espécies de peixes e provavelmente está relacionado às diferenças entre machos e fêmeas no que se refere ao processo de acúmulo de material de reserva nos gametas pois, como o gasto energético na produção de espermatozoides é menor, o macho pode produzi-los antes e estar sempre pronto para a fecundação.

Mesmo não havendo correlação significativa entre os valores do IGS e do IR, verificou-se que os estômagos dos espécimes examinados continham maior quantidade de alimento no período que antecedeu a desova. Isso sugere que $P$. bonariensis utiliza os recursos alimentares disponíveis no início da primavera (setembro e outubro, na região sul do Brasil) visando armazenar energia para o período reprodutivo (Nunes et al., 2011).

A análise da distribuição dos diâmetros dos oócitos de $P$. bonariensis no arroio Taquarembó mostrou uma distribuição polimodal, sugerindo desova do tipo parcelada sendo a mesma estratégia reprodutiva observada para essa espécie na represa de Yacyretá, na Argentina (Flores \& Hirt, 2002). Porém, no arroio Taquarembó essa espécie apresentou fecundidade média absoluta maior do que na referida represa (39.000 para 11.000) e o tamanho dos oócitos foram menores (539 mm para 839 $\mathrm{mm}$ ). Segundo NiKolsKiI (1969), a fecundidade pode ser ajustada automaticamente via metabolismo, que reage às mudanças no suprimento de alimento e produz alterações no número e no tamanho dos oócitos. A fecundidade relativa é um parâmetro importante da biologia reprodutiva, pois se refere ao número de oócitos que uma fêmea produz por unidade de peso do corpo, ou seja, seu investimento em prole reflete o estado físico das fêmeas podendo ser comparada entre espécies de diferentes tamanhos e pesos (Nikolskil, 1969; Bagenal, 1978). Não há dados sobre fecundidade relativa de $P$. bonariensis e nem de outras espécies do gênero. No arroio Taquarembó, $P$. bonariensis apresentou uma fecundidade relativa maior ( 0,43 oócitos/ $\mathrm{mg}$ ) se comparado ao cienídeo Plagioscion squamosissimus (Heckel, 1840), que teve os valores entre 0,013 e 0,108 oócitos/mg na represa Barra Bonita (BRAGA, 1997).

O início da maturidade sexual representa uma mudança crítica na vida dos indivíduos, pois a reprodução aumenta a demanda por recursos que antes eram utilizados somente para crescimento e sobrevivência (WoOtTon, 1991). No lago Guaíba e na laguna dos Patos o tamanho de primeira maturação para as fêmeas foi estimado em 178 mm de comprimento total (MARQUES et al., 2007), um valor aparentemente próximo ao obtido para as fêmeas da espécie no arroio Taquarembó (112,67 mm de comprimentopadrão), se consideradas as diferenças metodológicas. Diferenças no tamanho de primeira maturação podem ocorrer, segundo TARKAN (2006), em razão de que o comprimento máximo teórico alcançado pelos indivíduos entre espécies e populações pode variar dependendo do habitat que as mesmas ocupam. Mérona et al. (2009) verificaram uma diminuição no tamanho de primeira maturação em todas as espécies de peixes por eles estudados em dois reservatórios, após o represamento. É possível que isso também venha a ocorrer com a população de $P$. bonariensis do arroio Taquarembó. A proporção sexual, segundo NIKOLSKII (1969), é influenciada principalmente pelas condições ambientais e pela disponibilidade do alimento, que interferem no recrutamento, no crescimento e na mortalidade dos indivíduos. No estudo realizado na laguna dos Patos por Dufech \& Fialho (2007), a proporção sexual de $P$. bonariensis foi de aproximadamente cinco fêmeas para um macho. No arroio Taquarembó, o predomínio de fêmeas foi significativo apenas em alguns meses e classes de comprimento, mas não na população total.

Segundo NIKOLSKII (1963), variações no tamanho corporal entre os sexos ocorrem de maneira generalizada entre os peixes, normalmente com predomínio de fêmeas nas maiores classes de tamanho uma vez que a fecundidade está positivamente relacionada ao tamanho das mesmas (WoOtTon, 1991).

Winemiller \& Rose (1992) identificaram três estratégias de história de vida em peixes de água doce, quais sejam oportunistas, periódicas e de equilíbrio, e que representam trade-offs entre os parâmetros de sobrevivência, fecundidade e início e duração do período reprodutivo. Além disso, estas estratégias estariam em consonância com a variabilidade, previsibilidade e sazonalidade dos regimes ambientais. As características reprodutivas de $P$. bonariensis estão de acordo com a estratégia de história de vida do tipo periódica (sensu WINEMILLER \& Rose, 1992), uma vez que a espécie apresenta oócitos pelágicos e pequenos, com tamanho máximo de 524 mm, fecundidade elevada, ausência de cuidado parental, período reprodutivo curto e desova do tipo parcelada. As modificações no ambiente, como as advindas de construções de barragens, que mudam a dinâmica de um sistema natural e estabelecem novas condições ecológicas, podem alterar a estratégia reprodutiva de espécies de peixes para que estas se adequem às novas condições ambientais (MIMS \& OLDEN, 2013). Peixes são conhecidos pela grande plasticidade fenotípica com que respondem às características do meio, o que lhes permite aumentar as chances de sucesso frente a variações ambientais (Mérona et al., 2009). Segundo 
Spranza \& Stanley (2000), os peixes podem alterar sua fisiologia, sua estratégia reprodutiva e seu comportamento, proporcionando minimizarem custos e maximizarem benefícios associados à flutuação do ambiente, justamente por apresentarem uma alta plasticidade reprodutiva. Como exemplo disso, o cienídeo $P$. squamosissimus, da bacia do rio Paraná, teve seu sucesso reprodutivo atribuído à sua estratégia reprodutiva, caracterizada pela produção de pequenos ovos pelágicos liberados em vários lotes durante o período reprodutivo, além de apresentar larvas também pelágicas (Suzuki et al., 2005). Pachyurus bonariensis apresentou uma estratégia similar e esse poderá ser um fator favorável à sua adaptação ao futuro reservatório no arroio Taquarembó. Além disso, no local de estudo foram capturados indivíduos em todos os estádios de maturidade e com gônadas em pleno desenvolvimento, o que sugere que $P$. bonariensis é uma espécie sedentária ou residente naquele ambiente.

Como mudanças de ordem econômica, social e ambiental que ocorrerão no local de estudo após a construção desse represamento, os resultados aqui descritos poderão servir de base para avaliar principalmente os impactos que serão causados na população e na biologia reprodutiva dessa espécie no arroio Taquarembó.

Agradecimentos. A CAPES pela bolsa concedida; aos Drs. Luiz Roberto Malabarba, Lilian Casatti, Marco Aurélio Azevedo pelas críticas e sugestões; ao colega Carlos Eduardo B. Machado por ceder os exemplares aqui estudados e aos colegas do laboratório de Ictiologia/ UFRGS pelo auxílio durante a realização deste estudo.

\section{REFERÊNCIAS BIBLIOGRÁFICAS}

Adebisi, A. A. 1987. The relationships between fecundities, gonadossomatics indices and egg sizes of some fishes of Ogun River, Nigéria. Archiv für Hydrobiology 111(1):151-156.

Agostinho, A. A.; Julio Jr., H. F. \& Borghetti, J. R. 1992. Considerações sobre os impactos dos represamentos na ictiofauna e medidas para sua atenuação. Um estudo de caso: Reservatório de Itaipu. Revista Unimar 14(supl.):89-107.

Azevedo, M. A. 2010. Reproductive characteristics of characid fish species (Teleostei, Characiformes) and their relationship with body size and phylogeny. Iheringia, Série Zoologia 100(4):469-482.

Azevedo, M. A.; Malabarba, L. R. \& Fialho, C. B. 2000. Reproductive biology of the inseminated Glandulocaudine Diapoma speculiferum (Actinopterygii: Characidae). Copeia, 2000(4):983-989.

Bagenal, T. B. 1978. Methods for assessment of fish production in fresh waters. Oxford, Blackwell Scientific Publications. 365p.

Braga, F. M. DE S. 1997. Biologia reprodutiva de Plagioscion squamosissimus (Teleostei, Sciaenidae) na represa Barra Bonita, rio Piracicaba (SP), Revista UNIMAR 19(2):447-460.

Casatti, L. 2001. Taxonomia do gênero Sul-Americano Pachyurus Agassiz, 1831 (Teleostei: Perciformes: Sciaenidae) e descrição de duas novas espécies. Comunicações do Museu de Ciências e Tecnológicas da PUCRS, Série Zoologia 14(2):133-178.

Cecilio, E. B.; Agostinho, A. A.; Júlio Jr, H. F. \& Pavanelli, C. S. 1997. Colonização ictiofaunística do reservatório de Itaipu e áreas adjacentes. Revista Brasileira de Zoologia 14(1):1-14.

DufeCh. A. P. S. \& Fialho, C. B. 2007. Biologia populacional de Pachyurus bonariensis Steindachner, 1879 (Perciformes, Sciaenidae), uma espécie alóctone no sistema hidrográfico da laguna dos Patos, Brasil. Biota Neotropica 7(1):105-110.

Flores, S. A. \& HirT, L. M. 2002. Ciclo reprodutivo e Fecundidade de Pachyurus bonariensis (Steindachner, 1879) (Pisces, Sciaenidae). Boletim do Instituto de Pesca 28(1):25-31.
Giora, J. \& Fialho, C. B. 2009. Reproductive Biology of Weakly Electric Fish Eigenmanniatri lineata López and Castello, 1966 (Teleostei, Sternopygidae). Brazilian Archives of Biology and Technology 52(3):617-628.

Gonçalves, T. K.; Azevedo, M. A.; Malabarba, L. R. \& Fialho, C. B. 2005. Reproductive biology and development of sexually dimorphic structures in Aphyocharax anisitsi (Ostariophysi: Characidae). Neotropical Ichthyology 3(3):433-438.

Marques, C. da S.; Braun, A. S. \& Fontoura, N. F. 2007. Estimativa de tamanho de primeira maturação a partir de dados de IGS: Oligosarcus jenynsii, Oligosarcus robustus, Hoplias malabaricus, Cyphocharax voga, Astyanax fasciatus (Characiformes), Parapimelodus nigribarbis, Pimelodus maculatus, Trachelyopterus lucenai, Hoplosternum littorale, Loricariichthys anus (Siluriformes) e Pachyurus bonariensis (Perciformes) no lago Guaíba e laguna dos Patos, RS. Biociências 15(2):230-256.

Mérona, B. D. E.; Mol, J.; Vigouroux, R. \& Chaves, P.T. 2009. Phenotypic plasticity in fish life-history traits in two neotropical reservoirs: Petit-Saut Reservoir in French Guiana and Brokopondo Reservoir in Suriname. Neotropical Ichthyology 7(4):683-692.

Milani, P. C. C. \& Fontoura, N. F. 2007. Diagnóstico da pesca artesanal na Lagoa do Casamento, sistema nordeste da laguna dos Patos: uma proposta de manejo. Biociências 15(1):82-125.

Mims, M. C. \& OldEN, J. D. 2013. Fish assemblages respond to altered flow regimes via ecological filtering of life history strategies. Freshwater Biology 58:50-62.

Muniz, J. A. S. DE M.; Castanho, M. T. J. De A. \& Santos, A. J. G. Dos. 2008. Influência do fotoperíodo natural na reprodução induzida do tambaqui, Colossoma macropomum (Cuvier, 1818). Boletim Instituto Pesca 34(2):205-211.

Neuberger, A. L.; Marques, E. E.; Agostinho, C. S. \& Oliveira, R. J. De. 2007. Reproductive biology of Rhaphiodonvulpinus (Ostariophysi: Cynodontidae) in the Tocantins River Basin, Brazil. Neotropical Ichthyology 5(4):479-484.

Nikolskil, G. V. 1963. The ecology of fishes. London, Academic Press. 352p.

. 1969. Theory of fish population dynamics as the biological background for rational explotation and management of fishery resources. Edinburgh, Oliver \& Boyd. 323p.

Nunes, C.; Silva, A.; Soares, E. \& Ganias, K. 2011. The use of hepatic and somatic indices and histological information to characterize the reproductive dynamics of atlantic sardine Sardina pilchardus from the Portuguese coast. Marine and Coastal Fisheries: Dynamics, Management, and Ecosystem Science 3:127-144.

Pacheco, A. C. G.; Albrecht, M. P. \& Caramaschi, E. P. 2008. Ecologia de duas espécies de Pachyurus (Perciformes, Sciaenidae) do rio Tocantins, na região represada pela UHE Serra da Mesa, Goiás. Iheringia, Série Zoologia 98(2):270-277.

Saccol-Pereira, A. \& Fialho, C. B. 2010. Seasonal and diel variation in the fish assemblage of a Neotropical delta in southern Brazil. Iheringia, Série Zoologia 100(2):169-178.

Santin, M.; Bialetzki, A.; Assakawa, L. F. \& Taguti, T. L. 2009. Abundância e distribuição temporal de larvas de Pachyurus bonariensis Steindachner, 1879 (Perciformes, Sciaenidae), em uma baía do Pantanal matogrossense. Acta Scientiarum, Biological Sciences 31(1):65-71.

SAntos, E. P. DOS 1978. Dinâmica de populações aplicada à pesca e piscicultura. São Paulo, Edusp. 129p.

Santos, N. B.; Rocha, R. M. DA \& Fredóu, F. L. 2010. Reproductive biology of Plagioscion magdalenae (Teleostei Sciaenidae) (Steindachner, 1878) in the bay of Marajo, Amazon Estuary, Brazil. Neotropical Ichthyology 8(2):333-340.

SATo, Y. \& Godinho, H. P. 1988. A questão do tamanho de primeira maturação dos peixes de Três Marias, MG. In: Associação MineIRA de Aquicultura ed. Coletânea de resumos dos encontros da Associação Mineira de Aquicultura (AMA):1982-1987. Brasília, CODEVASF. p.93-94

Spranza, J. J. \& Stanley, E. H. 2000. Condition, growth, and reproductive styles of fishes exposed to different environmental regimes in a prairie drainage. Environmental Biology of Fishes 59:99-109. 
Suzuki, H. I.; Bulla, C. K.; Agostinho, A. A. \& Gomes, L. C. 2005. Estratégias reprodutivas de assembléias de peixes em reservatórios. In: Rodrigues, L.; Thomaz, S. M.; Agostinho, A. A. \& Gomes, L. C. eds. Biocenoses em reservatórios. São Carlos, RiMa. p.223-242.

TARKAN, A. S. 2006. Reproductive ecology of two cyprinid fishes in an oligotrophic lake near the southern limits of their distribution range. Ecology of Freshwater Fish 15:131-138.

VAzzoler, A. E. A. DE M. 1996. Biologia da reprodução de peixes teleósteos: teoria e prática. Maringá, Editora da Universidade. 169p.
Winemiller, K. O. \& Rose, K. A. 1992. Patterns of life-history diversification in North American fishes: implications for population regulation. Canadian Journal of Fisheries and Aquatic Sciences 49:2196-2218.

Wootton, R. J. 1991. Ecology of Teleost fishes. Chapman \& Hall, London. 404p.

ZAR, J. H. 1999. Biostatistical Analysis. Prentice-Hall, New Jersey. 663p. 